\title{
Robust Stability Criteria for T-S Fuzzy Systems with Time-Varying Delays via Nonquadratic Lyapunov-Krasovskii Functional Approach
}

\author{
Sung Hyun Kim \\ School of Electrical Engineering, University of Ulsan, Daehak-ro 93, Nam-Gu, Ulsan 680-749, Republic of Korea \\ Correspondence should be addressed to Sung Hyun Kim; ie222.kim@gmail.com
}

Received 17 May 2016; Revised 13 September 2016; Accepted 5 October 2016

Academic Editor: Olfa Boubaker

Copyright (C) 2016 Sung Hyun Kim. This is an open access article distributed under the Creative Commons Attribution License, which permits unrestricted use, distribution, and reproduction in any medium, provided the original work is properly cited.

This paper tackles the issue of stability analysis for uncertain T-S fuzzy systems with interval time-varying delays, especially based on the nonquadratic Lyapunov-Krasovskii functional (NLKF). To this end, this paper first provides a less conservative relaxation technique and then derives a relaxed robust stability criterion that enhances the interactions among delayed fuzzy subsystems. The effectiveness of our method is verified by two examples.

\section{Introduction}

Over the past few decades, Takagi-Sugeno (T-S) fuzzy model has attracted great attention since it can systematically represent nonlinear systems via a kind of interpolation method that connects smoothly some local linear systems based on fuzzy weighting functions [1]. In particular, the T-S fuzzy model has the advantage that it allows the well-established linear system theory to be applied to the analysis and synthesis of nonlinear systems. For this reason, the T-S fuzzy model has been a popular choice not only in consumer products but also in industrial processes (refer to [2] and references therein).

As well-known, time-delay phenomena are ubiquitous in practical engineering systems such as aircraft systems, biological systems, and chemical engineering system [3-5]. Recently, thus, the research on nonlinear systems with state delays has been an important issue in the stability analysis of T-S fuzzy systems. In the literature, there are two major research trends to deal with such systems: one focuses on decreasing computational burdens required to solve a set of conditions from the Lyapunov-Krasovskii functional (LKF) approach, and the other focuses on improving the solvability of delay-dependent stability conditions despite significant computational efforts. Strictly speaking, the first trend is mainly based on Jensen's inequality approach [6-11] and the second one is based on the free-weighing matrix approach [12-16].

Recently, it is recognized that the common quadratic Lyapunov function approach leads to overconservative performance for a large number of fuzzy rules $[17,18]$. For this reason, it is essential to tackle the issue of stability analysis in the light of the nonquadratic Lyapunov-Krasovskii functional (NLKF) [19-23]. However, to our best knowledge, up to now, little progress has been made toward using NLKFs for the stability analysis. Motivated by the above concern, this paper proposes a relaxed stability criterion for uncertain T-S fuzzy systems with interval time-varying delays, especially obtained by the NLKF approach. To this end, this paper offers a proper relaxation method that can enhance the interactions among delayed fuzzy subsystems. Further, it is worth noticing that Jensen's inequality, given in [24], is applicable only to the case where the internal matrix is constant, that is, to the case where the common quadratic Lyapunov-Krasovskii functional (CQLKF) is employed. Thus, this paper focuses more on exploring the second trend in the direction of reducing the conservatism that stems from the CQLKF approach, without resorting to any delay-decomposition method. In this sense, this paper provides two examples numerically to show the effectiveness of our method. 
The rest of the paper is organized as follows. Section 2 gives a mathematical description of the system considered here and presents a useful lemma. Section 3 presents the main result of this paper. Furthermore, through numerical examples, Section 4 shows the verification of our results. Finally, Section 5 makes the concluding remarks.

Notation. Throughout this paper, standard notions will be adopted. The notations $X \geq Y$ and $X>Y$ mean that $X-Y$ is positive semidefinite and positive definite, respectively. In symmetric block matrices, $(*)$ is used as an ellipsis for terms that are induced by symmetry. For a square matrix $\mathscr{X}, \mathrm{He}(\mathscr{X})$ denotes $\mathscr{X}+\mathscr{X}^{T}$, where $\mathscr{X}^{T}$ is the transpose of $\mathscr{X}$. The natation $\operatorname{Conv}(\cdot)$ denotes the convex hull; $\operatorname{col}\left(v_{1}, v_{2}, \ldots, v_{n}\right)=$ $\left[\begin{array}{llll}v_{1}^{T} & v_{2}^{T} & \cdots & v_{n}^{T}\end{array}\right]^{T}$ for any vector $v_{i} ; \operatorname{diag}(\mathscr{A}, \mathscr{B})$ denotes a diagonal matrix with diagonal entries $\mathscr{A}$ and $\mathscr{B}$; and $\mathbb{N}_{r}^{+}=$ $\{1,2, \ldots, r\}$. For any matrix $\mathcal{S}_{i}$ or $\mathcal{S}_{i j}$,

$$
\begin{aligned}
& {\left[\mathcal{S}_{i}\right]_{r}=\left[\begin{array}{llll}
\mathcal{S}_{1} & \mathcal{S}_{2} & \cdots & \mathcal{S}_{r}
\end{array}\right],} \\
& {\left[\mathcal{S}_{i j}\right]_{r \times r}=\left[\begin{array}{llll}
{\left[\mathcal{S}_{1 i}\right]_{r}^{T}} & {\left[\mathcal{S}_{2 i}\right]_{r}^{T}} & \cdots & {\left[\mathcal{S}_{r i}\right]_{r}^{T}}
\end{array}\right]^{T} .}
\end{aligned}
$$

All matrices, if their dimensions are not explicitly stated, are assumed to be compatible for algebraic operation.

\section{System Description and Preliminaries}

Consider the following uncertain T-S fuzzy system, which represents a class of nonlinear systems: for $i \in \mathbb{N}_{r}^{+}$,

Plant Rule i. IF $\eta_{1}(t)$ is $\mathscr{F}_{i 1}$ and $\cdots \eta_{s}(t)$ is $\mathscr{F}_{i s}$, THEN

$$
\begin{aligned}
& \dot{x}(t)=A_{i} x(t)+A_{d, i} x(t-d(t))+E_{i} p(t), \\
& q(t)=G_{i} x(t)+G_{d, i} x(t-d(t)), \\
& x(t)=\psi(t),
\end{aligned}
$$

$$
t \in\left[-d_{2}, 0\right]
$$

where $x(t) \in \mathbb{R}^{n_{x}}$ and $x(t-d(t)) \in \mathbb{R}^{n_{x}}$ denote the state and the delayed state, respectively; the initial condition $\psi(t)$ is a continuously differentiable vector-valued function; $\mathscr{F}_{i j}$ denotes a fuzzy set; $\eta_{i}(t)$ denotes the $i$ th premise variable; and $r$ denotes the number of IF-THEN rules. In (2), $p(t) \in \mathbb{R}^{n_{p}}$ and $q(t) \in \mathbb{R}^{n_{q}}$ are used to describe the structured feedback uncertainty such that $p(t)=\Delta(t) q(t)$ and $\Delta^{T}(t) \Delta(t) \leq I \in$ $\mathbb{R}^{n_{q} \times n_{q}}$. Further, the state delay $d(t)$ is assumed to be unknown and time-varying with known bounds as follows: $d_{1} \leq d(t) \leq$ $d_{2}$, where $d_{1}$ and $d_{2}$ are constant. Then, the overall T-S fuzzy model is inferred as follows:

$$
\begin{aligned}
\dot{x}(t)= & A\left(\Theta_{t}\right) x(t)+A_{d}\left(\Theta_{t}\right) x(t-d(t)) \\
& +E\left(\Theta_{t}\right) p(t), \\
q(t)= & G\left(\Theta_{t}\right) x(t)+G_{d}\left(\Theta_{t}\right) x(t-d(t)),
\end{aligned}
$$

where $A\left(\Theta_{t}\right)=\sum_{i=1}^{r} \theta_{i} A_{i}, A_{d}\left(\Theta_{t}\right)=\sum_{i=1}^{r} \theta_{i} A_{d, i}, E\left(\Theta_{t}\right)=$ $\sum_{i=1}^{r} \theta_{i} E_{i}, G\left(\Theta_{t}\right)=\sum_{i=1}^{r} \theta_{i} G_{i}$, and $G_{d}\left(\Theta_{t}\right)=\sum_{i=1}^{r} \theta_{i} G_{d, i}$ in which $\theta_{i}\left(=\theta_{i}(\eta(t))\right.$ denotes the normalized fuzzy weighting function for the $i$ th rule; $\eta(t)=\operatorname{col}\left(\eta_{1}(x(t)), \ldots, \eta_{s}(x(t))\right)$ denotes the premise variable vector; and $\Theta_{t}=\operatorname{col}\left(\theta_{1}, \ldots, \theta_{r}\right)$ belongs to

$$
\begin{aligned}
\mathbb{S}_{\Theta} & =\left\{\operatorname{col}\left(\theta_{1}, \ldots, \theta_{r}\right) \mid \sum_{i=1}^{r} \theta_{i}=1, \alpha_{i} \leq \theta_{i} \leq \beta_{i}, \forall i\right. \\
& \left.\in \mathbb{N}_{r}^{+}\right\} .
\end{aligned}
$$

Assumption 1. The fuzzy weighting functions $\theta_{i}$ are differentiable and $\dot{\Theta}_{t}=\operatorname{col}\left(\dot{\theta}_{1}, \ldots, \dot{\theta}_{r}\right)$ belongs to

$$
\begin{aligned}
\mathbb{S}_{\dot{\Theta}} & =\left\{\operatorname{col}\left(\dot{\theta}_{1}, \ldots, \dot{\theta}_{r}\right) \mid \sum_{i=1}^{r} \dot{\theta}_{i}=0, \varrho_{1, i} \leq \dot{\theta}_{i} \leq \varrho_{2, i}, \forall i\right. \\
& \left.\in \mathbb{N}_{r}^{+}\right\} .
\end{aligned}
$$

To simplify the notations, we use $\theta_{i}^{d_{1}}=\theta_{i}\left(\eta\left(t-d_{1}\right)\right)$ and $\theta_{i}^{d_{2}}=\theta_{i}\left(\eta\left(t-d_{2}\right)\right)$. And, for later convenience, we define $\bar{x}(t)=\operatorname{col}\left(x(t), x\left(t-d_{1}\right), x(t-d(t)), x\left(t-d_{2}\right)\right)$, $\eta(t)=\operatorname{col}(\bar{x}(t), p(t)) \in \mathbb{R}^{n_{\eta}}$, and $n_{\eta}=4 n_{x}+n_{p}$. And we use some block entry matrices $\mathbf{e}_{i}(i=1,2, \ldots, 5)$ such that $x(t)=\mathbf{e}_{1} \eta(t), x\left(t-d_{1}\right)=\mathbf{e}_{2} \eta(t), x(t-d(t))=\mathbf{e}_{3} \eta(t), x\left(t-d_{2}\right)=$ $\mathbf{e}_{4} \eta(t)$, and $p(t)=\mathbf{e}_{5} \eta(t)$, which implies $\bar{x}(t)=\mathbf{e}_{14} \eta(t)$ by defining $\mathbf{e}_{14}^{T}=\left[\begin{array}{lll}\mathbf{e}_{1}^{T} & \cdots & \mathbf{e}_{4}^{T}\end{array}\right]$. Then, (3) becomes

$$
\begin{aligned}
& \dot{x}(t)=\Phi_{t} \eta(t), \\
& q(t)=\Psi_{t} \eta(t),
\end{aligned}
$$

where $\Phi_{t}=A\left(\Theta_{t}\right) \mathbf{e}_{1}+A_{d}\left(\Theta_{t}\right) \mathbf{e}_{3}+E\left(\Theta_{t}\right) \mathbf{e}_{5}$ and $\Psi_{t}=G\left(\Theta_{t}\right) \mathbf{e}_{1}+$ $G_{d}\left(\Theta_{t}\right) \mathbf{e}_{3}$.

Lemma 2. Let $\Theta_{t} \in \mathbb{S}_{\Theta}$ be satisfied. Then, the following condition holds:

$$
\begin{aligned}
0> & \mathscr{M} \\
= & \mathscr{M}_{0}+\sum_{i=1}^{r} \theta_{i} \operatorname{He}\left(\mathscr{M}_{i}\right)+\sum_{i=1}^{r} \theta_{i}^{2} \mathscr{M}_{i i} \\
& +\sum_{i=1}^{r}\left(\sum_{j=i+1}^{r} \theta_{i} \theta_{j} \mathscr{M}_{i j}+\sum_{j=1}^{i-1} \theta_{i} \theta_{j} \mathscr{M}_{j i}^{T}\right)
\end{aligned}
$$

if there are all decision variables such that

$$
\begin{aligned}
& 0>\mathscr{L}=\left[\begin{array}{l|l}
\mathscr{L}_{0} & {\left[\mathscr{L}_{i}\right]_{r}} \\
\hline(*) & {\left[\mathscr{L}_{i j}\right]_{r r}}
\end{array}\right], \\
& 0<X_{i}+X_{i}^{T},
\end{aligned}
$$

$$
\forall i \in \mathbb{N}_{r}^{+}
$$

where $\mathscr{L}_{0}=\mathscr{M}_{0}+\mathrm{He}\left(S_{0}-\sum_{i=1}^{r} \alpha_{i} \beta_{i} X_{i}\right), \mathscr{L}_{i}=\mathscr{M}_{i}+S_{i}-S_{0}+$ $\left(\alpha_{i}+\beta_{i}\right) X_{i}, \mathscr{L}_{i i}=\mathscr{M}_{i i}+\mathrm{He}\left(-S_{i}-X_{i}\right)$, and $\mathscr{L}_{i j}=\mathscr{M}_{i j}-S_{i}-S_{j}$. 
Lemma 3. Let $\dot{\Theta}_{t} \in \mathbb{S}_{\dot{\Theta}}$ be satisfied. Then, the following condition holds:

$$
0>\Omega+\sum_{i=1}^{r} \dot{\theta}_{i} \mathscr{P}_{i}
$$

if there are all decision variables such that

$$
\begin{aligned}
& 0>\Omega+\sum_{i=1}^{r} Q_{\ell_{i},}\left(\mathscr{P}_{i}+Q\right), \\
& \forall \ell \in \mathbb{L}=\left\{\left(\ell_{1}, \ldots, \ell_{r}\right) \mid \ell_{i} \in \mathbb{N}_{2}^{+}, i \in \mathbb{N}_{r}^{+}\right\} .
\end{aligned}
$$

Proof. In view of $\dot{\Theta}_{t} \in \mathbb{S}_{\dot{\Theta}}$, we can get

$$
\begin{aligned}
\dot{\theta}_{i} & =\sum_{\ell_{\mathrm{i}}=1}^{2} \lambda_{\ell_{i}}(t) \varrho_{\ell_{i},}, \\
0 & =\sum_{i=1}^{r} \dot{\theta}_{i} \mathcal{N},
\end{aligned}
$$

where coefficients $\lambda_{\ell_{i}}$ are all positive and sum to one and $\mathcal{N}$ is a constant slack variable. Then, (9) leads to

$$
0>\Omega+\sum_{i=1}^{r}\left(\sum_{\ell_{i}=1}^{2} \lambda_{\ell_{i}}(t) \varrho_{\ell_{i}, i}\right)\left(\mathscr{P}_{i}+\mathcal{N}\right),
$$

which holds if (10) holds because $\sum_{\ell_{i}=1}^{2} \lambda_{\ell_{i}}(t) \varrho_{\ell_{i},}\left(\mathscr{P}_{i}+\mathscr{N}\right) \epsilon$ $\operatorname{Conv}\left(\varrho_{\ell_{i}, i}\left(\mathscr{P}_{i}+\mathcal{N}\right)\right)$, where $\ell_{i}$ denotes the $i$ th element of $\ell \in$ $\mathbb{L}$.

\section{3. $\Theta_{t}-$ Dependent Stability Criterion}

Based on a nonquadratic Lyapunov-Krasovskii functional (NLKF), this section provides a less conservative stability criterion. To this end, we first choose an NLKF of the following form:

$$
\begin{aligned}
V(t)= & V_{1}(t)+V_{2}(t)+V_{3}(t), \\
V_{1}(t)= & x^{T}(t) P\left(\Theta_{t}\right) x(t), \\
V_{2}(t)= & \int_{t-d_{1}}^{t} x^{T}(\alpha) Q_{1}\left(\Theta_{\alpha}\right) x(\alpha) d \alpha \\
& +\int_{t-d_{2}}^{t} x^{T}(\alpha) Q_{2}\left(\Theta_{\alpha}\right) x(\alpha) d \alpha, \\
V_{3}(t)= & \int_{-d_{1}}^{0} \int_{t+\alpha}^{t} \dot{x}^{T}(\beta) R_{1}\left(\Theta_{\beta}\right) \dot{x}(\beta) d \beta d \alpha \\
& +\int_{-d_{2}}^{-d_{1}} \int_{t+\alpha}^{t} \dot{x}^{T}(\beta) R_{2}\left(\Theta_{\beta}\right) \dot{x}(\beta) d \beta d \alpha,
\end{aligned}
$$

where $P\left(\Theta_{t}\right), Q_{1}\left(\Theta_{\alpha}\right), Q_{2}\left(\Theta_{\alpha}\right), R_{1}\left(\Theta_{\beta}\right)$, and $R_{2}\left(\Theta_{\beta}\right)$ are positive definite for all admissible grades. Then, the time derivative of each $V_{i}(t)$ along the trajectories of (6) is given by

$$
\begin{gathered}
\dot{V}_{1}(t)=\eta^{T}(t)\left(\operatorname{He}\left(\mathbf{e}_{1}^{T} P\left(\Theta_{t}\right) \Phi_{t}\right)+\mathbf{e}_{1}^{T} \dot{P}\left(\Theta_{t}\right) \mathbf{e}_{1}\right) \eta(t), \\
\dot{V}_{2}(t)=\eta^{T}(t)\left(\mathbf{e}_{1}^{T}\left(Q_{1}\left(\Theta_{t}\right)+Q_{2}\left(\Theta_{t}\right)\right) \mathbf{e}_{1}\right. \\
\left.-\mathbf{e}_{2}^{T} Q_{1}\left(\Theta_{t-d_{1}}\right) \mathbf{e}_{2}-\mathbf{e}_{4}^{T} Q_{2}\left(\Theta_{t-d_{2}}\right) \mathbf{e}_{4}\right) \eta(t), \\
\dot{V}_{3}(t)=\eta^{T}(t)\left(d_{1} \Phi_{t}^{T} R_{1}\left(\Theta_{t}\right) \Phi_{t}+\delta \Phi_{t}^{T} R_{2}\left(\Theta_{t}\right) \Phi_{t}\right) \\
\cdot \eta(t)-\int_{t-d_{1}}^{t} \dot{x}^{T}(\alpha) R_{1}\left(\Theta_{\alpha}\right) \dot{x}(\alpha) d \alpha \\
-\int_{t-d_{2}}^{t-d_{1}} \dot{x}^{T}(\alpha) R_{2}\left(\Theta_{\alpha}\right) \dot{x}(\alpha) d \alpha,
\end{gathered}
$$

which leads to

$$
\dot{V}(t)=\eta^{T}(t) \Pi_{1} \eta(t)+\mathcal{O}_{1}+\mathcal{O}_{2},
$$

where

$$
\begin{aligned}
\Pi_{1}= & H e\left(\mathbf{e}_{1}^{T} P\left(\Theta_{t}\right) \Phi_{t}\right) \\
& +\mathbf{e}_{1}^{T}\left(\dot{P}\left(\Theta_{t}\right)+Q_{1}\left(\Theta_{t}\right)+Q_{2}\left(\Theta_{t}\right)\right) \mathbf{e}_{1} \\
& -\mathbf{e}_{2}^{T} Q_{1}\left(\Theta_{t-d_{1}}\right) \mathbf{e}_{2}-\mathbf{e}_{4}^{T} Q_{2}\left(\Theta_{t-d_{2}}\right) \mathbf{e}_{4} \\
& +d_{1} \Phi_{t}^{T} R_{1}\left(\Theta_{t}\right) \Phi_{t}+\delta \Phi_{t}^{T} R_{2}\left(\Theta_{t}\right) \Phi_{t}, \\
\mathcal{O}_{1}= & -\int_{t-d_{1}}^{t} \dot{x}^{T}(\alpha) R_{1}\left(\Theta_{\alpha}\right) \dot{x}(\alpha) d \alpha, \quad \delta=d_{2}-d_{1}, \\
\mathcal{O}_{2}= & -\int_{t-d_{2}}^{t-d(t)} \dot{x}^{T}\left(\alpha_{1}\right) R_{2}\left(\Theta_{\alpha_{1}}\right) \dot{x}\left(\alpha_{1}\right) d \alpha_{1} \\
& -\int_{t-d(t)}^{t-d_{1}} \dot{x}^{T}\left(\alpha_{2}\right) R_{2}\left(\Theta_{\alpha_{2}}\right) \dot{x}\left(\alpha_{2}\right) d \alpha_{2} .
\end{aligned}
$$

Remark 4. Indeed, it is hard to directly use Jensen's inequality approach to obtain the upper bounds of $\mathcal{O}_{1}$ and $\mathcal{O}_{2}$ because $R_{1}\left(\Theta_{\alpha}\right)$ and $R_{2}\left(\Theta_{\alpha}\right)$ are set to be dependent on $\Theta_{\alpha}$, which motivates the present study.

Lemma 5. Suppose that there exist matrices $U_{0}\left(\Theta_{t}\right), U_{1}\left(\Theta_{t}\right)$, and $U_{2}\left(\Theta_{t}\right) \in \mathbb{R}^{4 n_{x} \times n_{x}}$ and symmetric matrices $0<P\left(\Theta_{t}\right)$, $\dot{P}\left(\Theta_{t}\right), 0<Q_{1}\left(\Theta_{t-d_{1}}\right), 0<Q_{2}\left(\Theta_{t-d_{2}}\right), 0<Q_{1}\left(\Theta_{t}\right), 0<$ $Q_{2}\left(\Theta_{t}\right), 0<R_{1}\left(\Theta_{\alpha}\right), 0<R_{2}\left(\Theta_{\alpha_{p}}\right), 0<R_{1}\left(\Theta_{t}\right), 0<R_{2}\left(\Theta_{t}\right) \epsilon$ $\mathbb{R}^{n_{x} \times n_{x}}, M_{0}\left(\Theta_{t}\right), M_{1}\left(\Theta_{t}\right)$, and $M_{2}\left(\Theta_{t}\right) \in \mathbb{R}^{4 n_{x} \times 4 n_{x}}$ such that

$$
\begin{aligned}
& 0>\Pi_{1}+\Pi_{2}+\Gamma_{p}, \quad \forall p \in \mathbb{N}_{2}^{+}, \\
& 0 \leq\left[\begin{array}{cc}
M_{0}\left(\Theta_{t}\right) & U_{0}\left(\Theta_{t}\right) \\
(*) & R_{1}\left(\Theta_{\alpha}\right)
\end{array}\right], \\
& 0 \leq\left[\begin{array}{cc}
M_{p}\left(\Theta_{t}\right) & U_{p}\left(\Theta_{t}\right) \\
(*) & R_{2}\left(\Theta_{\alpha_{p}}\right)
\end{array}\right],
\end{aligned}
$$


where

$$
\begin{aligned}
\Pi_{1} & =\operatorname{He}\left(\mathbf{e}_{1}^{T} P\left(\Theta_{t}\right) \Phi_{t}\right)+\mathbf{e}_{1}^{T}\left(\dot{P}\left(\Theta_{t}\right)+Q_{1}\left(\Theta_{t}\right)\right. \\
& \left.+Q_{2}\left(\Theta_{t}\right)\right) \mathbf{e}_{1}-\mathbf{e}_{2}^{T} Q_{1}\left(\Theta_{t-d_{1}}\right) \mathbf{e}_{2}-\mathbf{e}_{4}^{T} Q_{2}\left(\Theta_{t-d_{2}}\right) \mathbf{e}_{4} \\
& +d_{1} \Phi_{t}^{T} R_{1}\left(\Theta_{t}\right) \Phi_{t}+\delta \Phi_{t}^{T} R_{2}\left(\Theta_{t}\right) \Phi_{t}, \\
\Pi_{2} & =\Psi_{t}^{T} \Psi_{t}-\mathbf{e}_{5}^{T} \mathbf{e}_{5}, \\
\Gamma_{p} & =\operatorname{He}\left(\mathbf{e}_{14}^{T} U_{0}\left(\Theta_{t}\right)\left(\mathbf{e}_{1}-\mathbf{e}_{2}\right)+\mathbf{e}_{14}^{T} U_{1}\left(\Theta_{t}\right)\left(\mathbf{e}_{3}-\mathbf{e}_{4}\right)\right. \\
& \left.+\mathbf{e}_{14}^{T} U_{2}\left(\Theta_{t}\right)\left(\mathbf{e}_{2}-\mathbf{e}_{3}\right)\right)+d_{1} \mathbf{e}_{14}^{T} M_{0}\left(\Theta_{t}\right) \mathbf{e}_{14} \\
& +\delta \mathbf{e}_{14}^{T} M_{p}\left(\Theta_{t}\right) \mathbf{e}_{14} .
\end{aligned}
$$

Then, (6) is robustly asymptotically stable for $d_{1} \leq d(t) \leq d_{2}$.

Proof. First of all, by incorporating the following equalities into (15),

$$
\begin{aligned}
0= & \bar{x}^{T}(t) M_{0}\left(\Theta_{t}\right) \bar{x}(t)\left(d_{1}-\int_{t-d_{1}}^{t} d \alpha\right) \\
0= & \bar{x}^{T}(t) M_{1}\left(\Theta_{t}\right) \bar{x}(t)\left(\left(d_{2}-d(t)\right)-\int_{t-d_{2}}^{t-d(t)} d \alpha_{1}\right), \\
0= & \bar{x}^{T}(t) M_{2}\left(\Theta_{t}\right) \bar{x}(t)\left(\left(d(t)-d_{1}\right)-\int_{t-d(t)}^{t-d_{1}} d \alpha_{2}\right), \\
0= & 2 \bar{x}^{T}(t) U_{0}\left(\Theta_{t}\right)\left(\left(\mathbf{e}_{1}-\mathbf{e}_{2}\right) \eta(t)-\int_{t-d_{1}}^{t} \dot{x}(\alpha) d \alpha\right), \\
0= & 2 \bar{x}^{T}(t) U_{1}\left(\Theta_{t}\right) \\
& \cdot\left(\left(\mathbf{e}_{3}-\mathbf{e}_{4}\right) \eta(t)-\int_{t-d_{2}}^{t-d(t)} \dot{x}\left(\alpha_{1}\right) d \alpha_{1}\right) \\
0= & 2 \bar{x}^{T}(t) U_{2}\left(\Theta_{t}\right) \\
& \cdot\left(\left(\mathbf{e}_{2}-\mathbf{e}_{3}\right) \eta(t)-\int_{t-d(t)}^{t-d_{1}} \dot{x}\left(\alpha_{2}\right) d \alpha_{2}\right)
\end{aligned}
$$

we can get

$$
\dot{V}(t)=\eta^{T}(t)\left(\Pi_{1}+\bar{\Gamma}_{p}\right) \eta(t)+\overline{\mathcal{O}}_{1}+\overline{\mathcal{O}}_{2},
$$

where

$$
\begin{aligned}
\bar{\Gamma}_{p} & =\operatorname{He}\left(\mathbf{e}_{14}^{T} U_{0}\left(\Theta_{t}\right)\left(\mathbf{e}_{1}-\mathbf{e}_{2}\right)+\mathbf{e}_{14}^{T} U_{1}\left(\Theta_{t}\right)\left(\mathbf{e}_{3}-\mathbf{e}_{4}\right)\right. \\
& \left.+\mathbf{e}_{14}^{T} U_{2}\left(\Theta_{t}\right)\left(\mathbf{e}_{2}-\mathbf{e}_{3}\right)\right)+d_{1} \mathbf{e}_{14}^{T} M_{0}\left(\Theta_{t}\right) \mathbf{e}_{14} \\
& +\delta \mathbf{e}_{14}^{T}\left(\sum_{p=1}^{2} \lambda_{p}(t) M_{p}(t)\right) \mathbf{e}_{14}, \\
\overline{\mathcal{O}}_{1} & =-\int_{t-d_{1}}^{t}\left[\begin{array}{c}
\bar{x}(t) \\
\dot{x}(\alpha)
\end{array}\right]^{T} \\
& \cdot\left[\begin{array}{cc}
M_{0}\left(\Theta_{t}\right) & U_{0}\left(\Theta_{t}\right) \\
(*) & R_{1}\left(\Theta_{\alpha}\right)
\end{array}\right]\left[\begin{array}{c}
\bar{x}(t) \\
\dot{x}(\alpha)
\end{array}\right] d \alpha
\end{aligned}
$$

$$
\begin{aligned}
\overline{\mathcal{O}}_{2} & =-\int_{t-d_{2}}^{t-d(t)}\left[\begin{array}{c}
\bar{x}(t) \\
\dot{x}\left(\alpha_{1}\right)
\end{array}\right]^{T} \\
& \cdot\left[\begin{array}{cc}
M_{1}\left(\Theta_{t}\right) & U_{1}\left(\Theta_{t}\right) \\
(*) & R_{2}\left(\Theta_{\alpha_{1}}\right)
\end{array}\right]\left[\begin{array}{c}
\bar{x}(t) \\
\dot{x}\left(\alpha_{1}\right)
\end{array}\right] d \alpha_{1} \\
& -\int_{t-d(t)}^{t-d_{1}}\left[\begin{array}{c}
\bar{x}(t) \\
\dot{x}\left(\alpha_{2}\right)
\end{array}\right]^{T} \\
& \cdot\left[\begin{array}{cc}
M_{2}\left(\Theta_{t}\right) & U_{2}\left(\Theta_{t}\right) \\
(*) & R_{2}\left(\Theta_{\alpha_{2}}\right)
\end{array}\right]\left[\begin{array}{c}
\bar{x}(t) \\
\dot{x}\left(\alpha_{2}\right)
\end{array}\right] d \alpha_{2}
\end{aligned}
$$

in which $\lambda_{1}(t)=\left(d_{2}-d(t)\right) /\left(d_{2}-d_{1}\right)$ and $\lambda_{2}(t)=(d(t)-$ $\left.d_{1}\right) /\left(d_{2}-d_{1}\right)$. Next, the structured feedback uncertainty, given as $0 \leq q^{T}(t) q(t)-p^{T}(t) p(t)$, can be converted into $0 \leq$ $\eta^{T}(t)\left(\Psi_{t}^{T} \Psi_{t}-\mathbf{e}_{5}^{T} \mathbf{e}_{5}\right) \eta(t)$, which yields $\dot{V}(t) \leq \eta^{T}(t)\left(\Pi_{1}+\right.$ $\left.\Pi_{2}+\bar{\Gamma}_{p}\right) \eta(t)+\overline{\mathcal{O}}_{1}+\overline{\mathcal{O}}_{2}$. That is, the robust stability for (6) is assured by $0>\eta^{T}(t)\left(\Pi_{1}+\Pi_{2}+\bar{\Gamma}_{p}\right) \eta(t)+\overline{\mathscr{O}}_{1}+\overline{\mathcal{O}}_{2}$. Therefore, if (18) holds, then $\overline{\mathcal{O}}_{1}+\overline{\mathcal{O}}_{2} \leq 0$, and hence the robust stability criterion is given by (17) because $\sum_{p=1}^{2} \lambda_{p}(t) M_{p}(t) \epsilon$ $\operatorname{Conv}\left(M_{p}\left(\Theta_{t}\right)\right)$.

In the absence of uncertainties, the T-S fuzzy system becomes $\dot{x}(t)=\Phi_{t} \bar{x}(t)$, where $\Phi_{t}=A\left(\Theta_{t}\right) \mathbf{e}_{1}+A_{d}\left(\Theta_{t}\right) \mathbf{e}_{3}$. The following corollary presents the stability criterion for nominal T-S fuzzy systems with time-varying delays.

Corollary 6. Suppose that there exist matrices $U_{0}\left(\Theta_{t}\right)$, $U_{1}\left(\Theta_{t}\right)$, and $U_{2}\left(\Theta_{t}\right) \in \mathbb{R}^{4 n_{x} \times n_{x}}$ and symmetric matrices $0<$ $P\left(\Theta_{t}\right), \dot{P}\left(\Theta_{t}\right), 0<Q_{1}\left(\Theta_{t-d_{1}}\right), 0<Q_{2}\left(\Theta_{t-d_{2}}\right), 0<Q_{1}\left(\Theta_{t}\right)$, $0<Q_{2}\left(\Theta_{t}\right), 0<R_{1}\left(\Theta_{\alpha}\right), 0<R_{2}\left(\Theta_{\alpha_{p}}\right), 0<R_{1}\left(\Theta_{t}\right), 0<$ $R_{2}\left(\Theta_{t}\right) \in \mathbb{R}^{n_{x} \times n_{x}}, M_{0}\left(\Theta_{t}\right), M_{1}\left(\Theta_{t}\right)$, and $M_{2}\left(\Theta_{t}\right) \in \mathbb{R}^{4 n_{x} \times 4 n_{x}}$ such that

$$
\begin{aligned}
& 0>\Pi_{1}+\Gamma_{p}, \quad \forall p \in \mathbb{N}_{2}^{+}, \\
& 0 \leq\left[\begin{array}{cc}
M_{0}\left(\Theta_{t}\right) & U_{0}\left(\Theta_{t}\right) \\
(*) & R_{1}\left(\Theta_{\alpha}\right)
\end{array}\right], \\
& 0 \leq\left[\begin{array}{cc}
M_{p}\left(\Theta_{t}\right) & U_{p}\left(\Theta_{t}\right) \\
(*) & R_{2}\left(\Theta_{\alpha_{p}}\right)
\end{array}\right], \\
& \forall p \in \mathbb{N}_{2}^{+},
\end{aligned}
$$

where

$$
\begin{aligned}
\Pi_{1} & =\operatorname{He}\left(\mathbf{e}_{1}^{T} P\left(\Theta_{t}\right) \Phi_{t}\right)+\mathbf{e}_{1}^{T}\left(\dot{P}\left(\Theta_{t}\right)+Q_{1}\left(\Theta_{t}\right)\right. \\
& \left.+Q_{2}\left(\Theta_{t}\right)\right) \mathbf{e}_{1}-\mathbf{e}_{2}^{T} Q_{1}\left(\Theta_{t-d_{1}}\right) \mathbf{e}_{2}-\mathbf{e}_{4}^{T} Q_{2}\left(\Theta_{t-d_{2}}\right) \mathbf{e}_{4} \\
& +d_{1} \Phi_{t}^{T} R_{1}\left(\Theta_{t}\right) \Phi_{t}+\delta \Phi_{t}^{T} R_{2}\left(\Theta_{t}\right) \Phi_{t}
\end{aligned}
$$




$$
\begin{aligned}
\Gamma_{p} & =\operatorname{He}\left(\mathbf{e}_{14}^{T} U_{0}\left(\Theta_{t}\right)\left(\mathbf{e}_{1}-\mathbf{e}_{2}\right)+\mathbf{e}_{14}^{T} U_{1}\left(\Theta_{t}\right)\left(\mathbf{e}_{3}-\mathbf{e}_{4}\right)\right. \\
& \left.+\mathbf{e}_{14}^{T} U_{2}\left(\Theta_{t}\right)\left(\mathbf{e}_{2}-\mathbf{e}_{3}\right)\right)+d_{1} \mathbf{e}_{14}^{T} M_{0}\left(\Theta_{t}\right) \mathbf{e}_{14} \\
& +\delta \mathbf{e}_{14}^{T} M_{p}\left(\Theta_{t}\right) \mathbf{e}_{14} .
\end{aligned}
$$

Then, (6) without uncertainties is asymptotically stable for $d_{1} \leq$ $d(t) \leq d_{2}$.

Proof. The proof is omitted since it is analogous to the derivation of Lemma 5.

\section{LMI-Based Stability Criterion}

Based on Lemmas 2 and 3, to derive a finite number of solvable LMI conditions from (17), this paper simply sets all the decision variables to be of affine dependence on fuzzyweighting functions:

$$
\begin{aligned}
P\left(\Theta_{t}\right) & =\sum_{i=1}^{r} \theta_{i} P_{i}, \\
\dot{P}\left(\Theta_{t}\right) & =\sum_{i=1}^{r} \dot{\theta}_{i} P_{i}, \\
Q_{1}\left(\Theta_{t}\right) & =\sum_{i=1}^{r} \theta_{i} Q_{1, i}, \\
Q_{2}\left(\Theta_{t}\right) & =\sum_{i=1}^{r} \theta_{i} Q_{2, i}, \\
Q_{1}\left(\Theta_{t-d_{1}}\right) & =\sum_{q=1}^{r} \theta_{q}^{d_{1}} Q_{1, q}, \\
Q_{2}\left(\Theta_{t-d_{2}}\right) & =\sum_{\phi=1}^{r} \theta_{\phi}^{d_{2}} Q_{2, \phi}, \\
R_{2}\left(\Theta_{t}\right) & =\sum_{i=1}^{r} \theta_{i} U_{1, i}, \\
R_{1}\left(\Theta_{t}\right) & =\sum_{i=1}^{r} \theta_{i} R_{1, i}, \\
R_{2}\left(\Theta_{t}\right) & =\sum_{i=1}^{r} \theta_{i}^{\alpha_{p}} \theta_{i}^{\alpha} R_{2, i}, \\
R_{i}, i & \theta_{i} R_{2, i},
\end{aligned}
$$

Remark 7. As a way to improve the performance to be considered, we can increase the degree of polynomial dependence on fuzzy-weighting functions, as in [31-33] but this is outside of the intended scope of this paper.

Theorem 8. Let $\dot{\Theta}_{t} \in \mathbb{S}_{\dot{\Theta}}$ be satisfied. Suppose that there exist matrices $U_{0, i}, U_{1, i}, U_{2, i} \in \mathbb{R}^{4 n_{x} \times n_{x}}$ and $S_{0}, S_{i}, X_{i} \in \mathbb{R}^{n_{c} \times n_{c}}\left(n_{c}=\right.$ $\left.6 n_{x}+n_{p}+n_{q}\right)$, for $i \in \mathbb{N}_{r}^{+}$, symmetric matrices $N, 0<P_{i}$, $0<Q_{1, i}, 0<Q_{2, i}, 0<R_{1, i}$, and $0<R_{2, i} \in \mathbb{R}^{n_{x} \times n_{x}}$, for $i \in \mathbb{N}_{r}^{+}$, and $M_{0, i}, M_{1, i}, M_{2, i} \in \mathbb{R}^{4 n_{x} \times 4 n_{x}}$ such that, for all $q, \phi \in \mathbb{N}_{r}^{+}$, $p \in \mathbb{N}_{2}^{+}$, and $\ell \in \mathbb{L}$,

$$
\begin{aligned}
& 0>\left[\begin{array}{c|c}
\mathscr{L}_{\ell q \phi, 0} \mid\left[\mathscr{L}_{p, i}\right]_{r} \\
\hline(*) & {\left[\mathscr{L}_{i j}\right]_{r \times r}}
\end{array}\right], \\
& 0<X_{q}+X_{q}^{T}, \\
& 0 \leq\left[\begin{array}{cc}
M_{0, \phi} & U_{0, \phi} \\
(*) & R_{1, q}
\end{array}\right], \\
& 0 \leq\left[\begin{array}{cc}
M_{p, \phi} & U_{p, \phi} \\
(*) & R_{2, q}
\end{array}\right],
\end{aligned}
$$

where $\mathscr{L}_{\text {eq }, 0}=\mathscr{M}_{\ell q \phi, 0}+\operatorname{He}\left(S_{0}-\sum_{i=1}^{r} \alpha_{i} \beta_{i} X_{i}\right), \mathscr{L}_{p, i}=\mathscr{M}_{p, i}+$ $S_{i}-S_{0}+\left(\alpha_{i}+\beta_{i}\right) X_{i}, \mathscr{L}_{i i}=\mathscr{M}_{i i}+\operatorname{He}\left(-S_{i}-X_{i}\right)$, and $\mathscr{L}_{i j}=$ $\mathscr{M}_{i j}-S_{i}-S_{j}$ in which

$$
\begin{aligned}
\mathscr{M}_{\ell q \phi, 0}= & \operatorname{diag}\left(-I, 0,0,(4,4)_{\ell q \phi, 0}\right), \\
\mathscr{M}_{p, i}= & {\left[\begin{array}{cccc}
0 & 0 & 0 & (1,4)_{i} \\
0 & -\frac{1}{2} \delta R_{2, i} & 0 & 0 \\
0 & 0 & -\frac{1}{2} d_{1} R_{1, i} & 0 \\
0 & 0 & 0 & (4,4)_{p, i}
\end{array}\right], } \\
\mathscr{M}_{i i}= & {\left[\begin{array}{cccc}
0 & 0 & 0 & 0 \\
0 & 0 & 0 & (2,4)_{i i} \\
0 & 0 & 0 & (3,4)_{i i} \\
0 & (*) & (*) & \mathrm{He}\left((4,4)_{i i}\right)
\end{array}\right], }
\end{aligned}
$$




$$
\begin{aligned}
\mathcal{M}_{i j}= & {\left[\begin{array}{lllc}
0 & 0 & 0 & 0 \\
0 & 0 & 0 & (2,4)_{i j}+(2,4)_{j i} \\
0 & 0 & 0 & (3,4)_{i j}+(3,4)_{j i} \\
0 & 0 & 0 & (4,4)_{i j}+(4,4)_{j i}
\end{array}\right], } \\
(4,4)_{\ell q \phi, 0}= & \mathbf{e}_{1}^{T}\left(\sum_{i=1}^{r} Q_{\ell_{i}, i}\left(P_{i}+N\right)\right) \mathbf{e}_{1}-\mathbf{e}_{2}^{T} Q_{1, q} \mathbf{e}_{2} \\
& -\mathbf{e}_{4}^{T} Q_{2, \phi} \mathbf{e}_{4}-\mathbf{e}_{5}^{T} \mathbf{e}_{5}, \\
(1,4)_{i}= & G_{i} \mathbf{e}_{1}+G_{d, i} \mathbf{e}_{3}, \\
(4,4)_{p, i}= & \mathbf{e}_{1}^{T}\left(\frac{1}{2} Q_{1, i}+\frac{1}{2} Q_{2, i}\right) \mathbf{e}_{1}+\Gamma_{p, i}, \\
(2,4)_{i j}= & \delta\left(R_{2, j} A_{i} \mathbf{e}_{1}+R_{2, j} A_{d, i} \mathbf{e}_{3}+R_{2, j} E_{i} \mathbf{e}_{5}\right), \\
(3,4)_{i j}= & d_{1}\left(R_{1, j} A_{i} \mathbf{e}_{1}+R_{1, j} A_{d, i} \mathbf{e}_{3}+R_{1, j} E_{i} \mathbf{e}_{5}\right), \\
(4,4)_{i j}= & \mathbf{e}_{1}^{T} P_{j} A_{i} \mathbf{e}_{1}+\mathbf{e}_{1}^{T} P_{j} A_{d, i} \mathbf{e}_{3}+\mathbf{e}_{1} P_{j} E_{i} \mathbf{e}_{5}, \\
\Gamma_{p, i}= & \mathbf{e}_{14}^{T} U_{0, i}\left(\mathbf{e}_{1}-\mathbf{e}_{2}\right)+\mathbf{e}_{14}^{T} U_{1, i}\left(\mathbf{e}_{3}-\mathbf{e}_{4}\right) \\
& +\mathbf{e}_{14}^{T} U_{2, i}\left(\mathbf{e}_{2}-\mathbf{e}_{3}\right)+\frac{1}{2} d_{1} \mathbf{e}_{14}^{T} M_{0, i} \mathbf{e}_{14} \\
& +\frac{1}{2} \delta \mathbf{e}_{14}^{T} M_{p, i} \mathbf{e}_{14} .
\end{aligned}
$$

Then, the system in (6) is robustly asymptotically stable for $d_{1} \leq$ $d(t) \leq d_{2}$.

Proof. Note that $\dot{\Theta}_{t} \in \mathbb{S}_{\dot{\Theta}}$. Thus, in view of Lemma 3, applying the Schur complement to (17) is given by

$$
\begin{array}{r}
0>\left[\begin{array}{ccc|c}
-I & 0 & 0 & \Psi_{t} \\
0 & -\delta R_{2}\left(\Theta_{t}\right) & 0 & \delta R_{2}\left(\Theta_{t}\right) \Phi_{t} \\
0 & 0 & -d_{1} R_{1}\left(\Theta_{t}\right) & d_{1} R_{1}\left(\Theta_{t}\right) \Phi_{t} \\
\hline(*) & (*) & (*) & \Omega_{\ell}+\Gamma_{p}
\end{array}\right], \\
\forall p \in \mathbb{N}_{2}^{+}, \ell \in \mathbb{L},
\end{array}
$$

where $\Omega_{\ell}=\operatorname{He}\left(\mathbf{e}_{1}^{T} P\left(\Theta_{t}\right) \Phi_{t}\right)-\mathbf{e}_{2}^{T} Q_{1}\left(\Theta_{t-d_{1}}\right) \mathbf{e}_{2}-\mathbf{e}_{4}^{T} Q_{2}\left(\Theta_{t-d_{2}}\right) \mathbf{e}_{4}$ $-\mathbf{e}_{5}^{T} \mathbf{e}_{5}+\mathbf{e}_{1}^{T}\left(\sum_{i=1}^{r} \varrho_{\ell_{i}, i}\left(P_{i}+N\right)+Q_{1}\left(\Theta_{t}\right)+Q_{2}\left(\Theta_{t}\right)\right) \mathbf{e}_{1}$. Further, from (26) and (27), (35) and (18) can be converted into

$$
\begin{aligned}
& 0>\sum_{q=1}^{r} \sum_{\phi=1}^{r} \theta_{q}^{d_{1}} \theta_{\phi}^{d_{2}} \mathscr{M}_{p, \ell q \phi}\left(\Theta_{t}\right) \in \mathbb{R}^{n_{c} \times n_{c}}, \\
& \qquad \leq p \in \mathbb{N}_{2}^{+}, \ell \in \mathbb{L}, \\
& 0 \leq \sum_{\phi=1}^{r} \sum_{q=1}^{r} \theta_{\phi} \theta_{q}^{\alpha}\left[\begin{array}{cc}
M_{0, \phi} & U_{0, \phi} \\
(*) & R_{1, q}
\end{array}\right], \\
& \sum_{\phi=1}^{r} \theta_{\phi} \theta_{q}^{\alpha_{p}}\left[\begin{array}{cc}
M_{p, \phi} & U_{p, \phi} \\
(*) & R_{2, q}
\end{array}\right],
\end{aligned}
$$

where

$$
\begin{aligned}
& \mathscr{M}_{p, \ell q \phi}\left(\Theta_{t}\right) \\
& =\left[\begin{array}{ccc|c}
-I & 0 & 0 & \Psi_{t} \\
0 & -\delta R_{2}\left(\Theta_{t}\right) & 0 & \delta R_{2}\left(\Theta_{t}\right) \Phi_{t} \\
0 & 0 & -d_{1} R_{1}\left(\Theta_{t}\right) & d_{1} R_{1}\left(\Theta_{t}\right) \Phi_{t} \\
\hline(*) & (*) & (*) & \Omega_{\ell q \phi}+\Gamma_{p}
\end{array}\right], \\
& \Omega_{\ell q \phi} \\
& =\operatorname{He}\left(\mathbf{e}_{1}^{T} P\left(\Theta_{t}\right) \Phi_{t}\right)-\mathbf{e}_{2}^{T} Q_{1, q} \mathbf{e}_{2}-\mathbf{e}_{4}^{T} Q_{2, \phi} \mathbf{e}_{4}-\mathbf{e}_{5}^{T} \mathbf{e}_{5} \\
& \quad+\mathbf{e}_{1}^{T}\left(\sum_{i=1}^{r} \varrho_{\ell_{i}, i}\left(P_{i}+N\right)+Q_{1}\left(\Theta_{t}\right)+Q_{2}\left(\Theta_{t}\right)\right) \mathbf{e}_{1} .
\end{aligned}
$$

As a result, from the convexity of fuzzy-weighting functions, (17) and (18) can be assured by (30),

$$
0>\mathscr{M}_{p, \ell q \phi}\left(\Theta_{t}\right), \quad \forall q, \phi \in \mathbb{N}_{r}^{+}, p \in \mathbb{N}_{2}^{+}, \ell \in \mathbb{L} .
$$

Further, note that representing (38) in the form of (7) becomes

$$
\begin{array}{r}
0>\mathscr{M}_{\ell q \phi, 0}+\sum_{i=1}^{r} \theta_{i} \operatorname{He}\left(\mathscr{M}_{p, i}\right)+\sum_{i=1}^{r} \theta_{i}^{2} \mathscr{M}_{i i} \\
+\sum_{i=1}^{r}\left(\sum_{j=i+1}^{r} \theta_{i} \theta_{j} \mathscr{M}_{i j}+\sum_{j=1}^{i-1} \theta_{i} \theta_{j} \mathscr{M}_{j i}^{T}\right),
\end{array}
$$

where $\mathscr{M}_{\ell q \phi, 0}, \mathscr{M}_{p, i}, \mathscr{M}_{i i}$, and $\mathscr{M}_{i j}$ are defined in (31)-(33). Therefore, from Lemma 2 , we can obtain (29) in the sequel without loss of generality.

The following corollary presents the LMI-based stability criterion for nominal T-S fuzzy systems with time-varying delays.

Corollary 9. Let $\dot{\Theta}_{t} \in \mathbb{S}_{\dot{\Theta}}$ be satisfied. Suppose that there exist matrices $U_{0, i}, U_{1, i}, U_{2, i} \in \mathbb{R}^{4 n_{x} \times n_{x}}$ and $S_{0}, S_{i}, X_{i} \in \mathbb{R}^{n_{c} \times n_{c}}\left(n_{c}=\right.$ $\left.6 n_{x}\right)$, for $i \in \mathbb{N}_{r}^{+}$, symmetric matrices $N, 0<P_{i}, 0<Q_{1, i}$, $0<Q_{2, i}, 0<R_{1, i}$, and $0<R_{2, i} \in \mathbb{R}^{n_{x} \times n_{x}}$, for $i \in \mathbb{N}_{r}^{+}$, and $M_{0, i}, M_{1, i}, M_{2, i} \in \mathbb{R}^{4 n_{x} \times 4 n_{x}}$ such that, for all $q, \phi \in \mathbb{N}_{r}^{+}, p \in \mathbb{N}_{2}^{+}$, and $\ell \in \mathbb{L}$,

$$
\begin{aligned}
& 0>\left[\begin{array}{c|c}
\mathscr{L}_{\text {eq }, 0} \mid\left[\mathscr{L}_{p, i}\right]_{r} \\
\hline(*) & {\left[\mathscr{L}_{i j}\right]_{r \times r}}
\end{array}\right], \\
& 0<X_{q}+X_{q}^{T}, \\
& 0 \leq\left[\begin{array}{cc}
M_{0, \phi} & U_{0, \phi} \\
(*) & R_{1, q}
\end{array}\right], \\
& 0 \leq\left[\begin{array}{cc}
M_{p, \phi} & U_{p, \phi} \\
(*) & R_{2, q}
\end{array}\right],
\end{aligned}
$$


where $\mathscr{L}_{\ell p \phi, 0}=\mathscr{M}_{\ell p \phi, 0}+\operatorname{He}\left(S_{0}-\sum_{i=1}^{r} \alpha_{i} \beta_{i} X_{i}\right), \mathscr{L}_{p, i}=\mathscr{M}_{p, i}+$ $S_{i}-S_{0}+\left(\alpha_{i}+\beta_{i}\right) X_{i}, \mathscr{L}_{i i}=\mathscr{M}_{i i}+\operatorname{He}\left(-S_{i}-X_{i}\right)$, and $\mathscr{L}_{i j}=$ $\mathscr{M}_{i j}-S_{i}-S_{j}$ in which

$$
\begin{aligned}
& \mathscr{M}_{\ell p \phi, 0}=\operatorname{diag}\left(0,0,(3,3)_{\ell p \phi, 0}\right) \\
& \mathscr{M}_{p, i}=\left[\begin{array}{ccc}
-\frac{1}{2} \delta R_{2, i} & 0 & 0 \\
0 & -\frac{1}{2} d_{1} R_{1, i} & 0 \\
0 & 0 & (3,3)_{p, i}
\end{array}\right] \text {, } \\
& \mathscr{M}_{i i}=\left[\begin{array}{ccc}
0 & 0 & (1,3)_{i i} \\
0 & 0 & (2,3)_{i i} \\
(*) & (*) & H e\left((3,3)_{i i}\right)
\end{array}\right], \\
& \mathscr{M}_{i j}=\left[\begin{array}{lll}
0 & 0 & (1,3)_{i j}+(1,3)_{j i} \\
0 & 0 & (2,3)_{i j}+(2,3)_{j i} \\
0 & 0 & (3,3)_{i j}+(3,3)_{j i}
\end{array}\right], \\
& (3,3)_{\ell q \phi, 0}=\mathbf{e}_{1}^{T}\left(\sum_{i=1}^{r} \varrho_{\ell_{i}, i}\left(P_{i}+N\right)\right) \mathbf{e}_{1}-\mathbf{e}_{2}^{T} Q_{1, q} \mathbf{e}_{2} \\
& -\mathbf{e}_{4}^{T} Q_{2, \phi} \mathbf{e}_{4} \\
& (3,3)_{p, i}=\mathbf{e}_{1}^{T}\left(\frac{1}{2} Q_{1, i}+\frac{1}{2} Q_{2, i}\right) \mathbf{e}_{1}+\Gamma_{p, i}, \\
& (1,3)_{i j}=\delta\left(R_{2, j} A_{i} \mathbf{e}_{1}+R_{2, j} A_{d, i} \mathbf{e}_{3}\right), \\
& (2,3)_{i j}=d_{1}\left(R_{1, j} A_{i} \mathbf{e}_{1}+R_{1, j} A_{d, i} \mathbf{e}_{3}\right), \\
& (3,3)_{i j}=\mathbf{e}_{1}^{T} P_{j} A_{i} \mathbf{e}_{1}+\mathbf{e}_{1}^{T} P_{j} A_{d, i} \mathbf{e}_{3}, \\
& \Gamma_{p, i}=\mathbf{e}_{14}^{T} U_{0, i}\left(\mathbf{e}_{1}-\mathbf{e}_{2}\right)+\mathbf{e}_{14}^{T} U_{1, i}\left(\mathbf{e}_{3}-\mathbf{e}_{4}\right) \\
& +\mathbf{e}_{14}^{T} U_{2, i}\left(\mathbf{e}_{2}-\mathbf{e}_{3}\right)+\frac{1}{2} d_{1} \mathbf{e}_{14}^{T} M_{0, i} \mathbf{e}_{14} \\
& +\frac{1}{2} \delta \mathbf{e}_{14}^{T} M_{p, i} \mathbf{e}_{14}
\end{aligned}
$$

Then, (6) without uncertainties is asymptotically stable for $d_{1} \leq$ $d(t) \leq d_{2}$.

Proof. The proof is omitted since it is analogous to the derivation of Theorem 8 .

Remark 10. The number of scalar variables involved in Theorem 8 and Corollary 9 is given as follows: $\#=n_{c}^{2}(2 r+1)+$ $0.5 n_{x}\left(n_{x}+1\right)+n_{x}\left(38.5 n_{x}+8.5\right) r$. Table 1 shows the number for each case of $\left(n_{x}, r\right)$. Since the use of slack variables requires more computation cost compared with other methods, there may be the need to balance the tradeoffs between the computational cost and the performance enhancement.

\section{Numerical Examples}

To verify the effectiveness of our methods, this paper provides two examples that make some comparisons with other results: one is related to the stability analysis for nominal T-S fuzzy systems and the other is related to the robust stability analysis for T-S fuzzy systems with uncertainties.

Example 1. Consider the following T-S fuzzy system, adopted in [25]:

$$
\begin{aligned}
A_{1} & =\left[\begin{array}{cc}
-2 & 0 \\
0 & -0.9
\end{array}\right], \\
A_{2} & =\left[\begin{array}{cc}
-1 & 0.5 \\
0 & -1
\end{array}\right], \\
A_{d, 1} & =\left[\begin{array}{cc}
-1 & 0 \\
-1 & -1
\end{array}\right], \\
A_{d, 2} & =\left[\begin{array}{cc}
-1 & 0 \\
0.1 & -1
\end{array}\right],
\end{aligned}
$$

where $\theta_{1}=1 /\left(1+\exp \left(-2 x_{1}(t)\right)\right)$ and $\theta_{2}=1-\theta_{1}$. Table 2 shows the maximum allowable upper bound (MAUB) for each $d_{1} \in\{0.0,0.4,0.8,1.0,1.2\}$, where $m$ denotes the number of delay segments and $(m-1)$ denotes the degree of delay partitioning. From Table 2, we can see that our method (Corollary 9) provides larger MAUBs in comparison with those of $[25,26]$. Hence it can be concluded that the stability criterion in Corollary 9, obtained based on the NLKF, is less conservative than other results. In particular, for $d_{1}=1.2$ and $d_{2}=1.531$, Corollary 9 offers the following solutions:

$$
\begin{aligned}
P_{1} & =10^{-2}\left[\begin{array}{cc}
1.485 & -0.227 \\
-0.227 & 0.368
\end{array}\right], \\
P_{2} & =10^{-2}\left[\begin{array}{cc}
1.078 & -0.290 \\
-0.290 & 0.799
\end{array}\right] \\
Q_{1,1} & =10^{-3}\left[\begin{array}{cc}
3.315 & 0.253 \\
0.253 & 0.785
\end{array}\right] \\
Q_{1,2} & =10^{-3}\left[\begin{array}{cc}
3.082 & 0.163 \\
0.163 & 0.930
\end{array}\right] \\
Q_{2,1} & =10^{-3}\left[\begin{array}{cc}
5.607 & 0.254 \\
0.254 & 3.137
\end{array}\right] \\
Q_{2,2} & =10^{-3}\left[\begin{array}{cc}
5.708 & -0.001 \\
-0.001 & 3.323
\end{array}\right] \\
R_{1,1} & =10^{-3}\left[\begin{array}{cc}
4.661 & -0.847 \\
-0.847 & 1.669
\end{array}\right] \\
R_{1,2} & =10^{-3}\left[\begin{array}{cc}
4.647 & -0.813 \\
-0.813 & 1.671
\end{array}\right]
\end{aligned}
$$


TABLE 1: \# involved in Corollary 9 and Theorem $8\left(n_{p}=1, n_{q}=1\right)$.

\begin{tabular}{llllllllll}
\hline$\left(n_{x}, r\right)$ & $(2,2)$ & $(2,3)$ & $(2,4)$ & $(3,2)$ & $(3,3)$ & $(3,4)$ & $(4,2)$ & $(4,3)$ & $(4,4)$ \\
\hline Corollary 9 & 1065 & 1524 & 1983 & 2370 & 3390 & 4410 & 4190 & 5992 & 7794 \\
Theorem 8 & 1325 & 1888 & 2451 & 2750 & 3922 & 5094 & 4690 & 6692 & 8694 \\
\hline
\end{tabular}

TABLE 2: Maximum allowable upper bound (MAUB) for each $d_{1}$, where $m$ denotes the number of delay segments and $(m-1)$ denotes the degree of delay partitioning.

\begin{tabular}{lcccccc}
\hline$d_{1}$ & 0.0 & 0.4 & 0.8 & 1.0 & & 1.2 \\
\hline$[25]$ & 0.982 & 1.038 & 1.158 & 1.252 & 1.359 & 0 \\
{$[26]$} & 1.221 & 1.277 & 1.311 & 1.358 & 1.419 & 1 \\
{$[26]$} & 1.278 & 1.303 & 1.316 & 1.361 & 1.425 & 2 \\
Corollary 9 & 1.302 & 1.380 & 1.413 & 1.462 & 1.531 & 0 \\
\hline
\end{tabular}

TABLE 3: Maximum allowable upper bound (MAUB) for $d_{1}=0$.

\begin{tabular}{lcccccc}
\hline Methods & {$[27]$} & {$[28]$} & {$[29]$} & {$[30]$} & Theorem 8 \\
\hline$d_{2}$ & - & 0.443 & 0.499 & 1.081 & 1.132 \\
\hline
\end{tabular}

$$
\begin{aligned}
& R_{2,1}=10^{-3}\left[\begin{array}{cc}
7.232 & -1.319 \\
-1.319 & 2.747
\end{array}\right], \\
& R_{2,2}=10^{-3}\left[\begin{array}{cc}
6.538 & -0.704 \\
-0.704 & 2.583
\end{array}\right]
\end{aligned}
$$

Example 2. Consider the following T-S fuzzy system:

$$
\begin{aligned}
A_{1} & =\left[\begin{array}{cc}
-2 & 1 \\
0.5 & -1
\end{array}\right], \\
A_{2} & =\left[\begin{array}{cc}
-2 & 0 \\
0 & -1
\end{array}\right], \\
A_{d, 1} & =\left[\begin{array}{cc}
-1 & 0 \\
-1 & -1
\end{array}\right], \\
A_{d, 2} & =\left[\begin{array}{cc}
-1.6 & 0 \\
0 & -1
\end{array}\right], \\
E_{1} & =\left[\begin{array}{cc}
0.03 & 0 \\
0 & -0.03
\end{array}\right], \\
E_{2} & =\left[\begin{array}{cc}
0.03 & 0 \\
0 & -0.03
\end{array}\right], \\
G_{1} & =\left[\begin{array}{cc}
1.6 & 0 \\
0 & 0.05
\end{array}\right], \\
G_{2} & =\left[\begin{array}{cc}
1.6 & 0 \\
0 & -0.05
\end{array}\right],
\end{aligned}
$$

$$
\begin{aligned}
& G_{d 1}=\left[\begin{array}{cc}
0.1 & 0 \\
0 & 0.3
\end{array}\right], \\
& G_{d 2}=\left[\begin{array}{cc}
0.1 & 0 \\
0 & 0.3
\end{array}\right],
\end{aligned}
$$

where

$$
\begin{aligned}
\theta_{1}= & \left(1-\frac{1}{1+\exp \left(-6\left(x_{2}-\pi / 4\right)\right)}\right) \\
& \times\left(\frac{1}{1+\exp \left(-6\left(x_{2}+\pi / 4\right)\right)}\right), \\
\theta_{2}= & 1-\theta_{1} .
\end{aligned}
$$

The maximum allowable upper bound (MAUB) for each method is tabulated in Table 3. And, from Table 3, we can see that the proposed method (Theorem 8) achieves larger MAUBs than those of other methods [27-30]. Hence, it can be concluded that the robust stability criterion in Theorem 8 , established from the NLKF approach and Lemma 2, is less conservative than those of [27-30].

\section{Concluding Remarks}

This paper proposed an NLKF-based method of deriving a less conservative stability criterion for T-S fuzzy systems with time-varying delays. Of course, the proposed method may increase the burden of numerical computation. However, if the computational complexity is out of the practical problem, then our results can be significantly useful.

\section{Competing Interests}

The author declares that there is no conflict of interests regarding the publication of this paper. 


\section{Acknowledgments}

This work was supported by the National Research Foundation of Korea Grant funded by the Korean Government (NRF-2015R1A1A1A05001131).

\section{References}

[1] T. Takagi and M. Sugeno, "Fuzzy identification of systems and its applications to modeling and control," IEEE Transactions on Systems, Man and Cybernetics, vol. 15, no. 1, pp. 116-132, 1985.

[2] G. Feng, "A survey on analysis and design of model-based fuzzy control systems," IEEE Transactions on Fuzzy Systems, vol. 14, no. 5, pp. 676-697, 2006.

[3] J. Cheng, H. Wang, S. Chen, Z. Liu, and J. Yang, "Robust delay-derivative-dependent state-feedback control for a class of continuous-time system with time-varying delays," Neurocomputing, vol. 173, pp. 827-834, 2016.

[4] Y. Ren, Z. Feng, and G. Sun, "Improved stability conditions for uncertain neutral-type systems with time-varying delays," International Journal of Systems Science, vol. 47, no. 8, pp. 1982-1993, 2016.

[5] S. H. Kim, "Relaxed inequality approach to robust $H_{\infty}$ stability analysis of discrete-time systems with time-varying delay," IET Control Theory \& Applications, vol. 6, no. 13, pp. 2149-2156, 2012.

[6] C.-H. Lien and K.-W. Yu, "Robust control for Takagi-Sugeno fuzzy systems with time-varying state and input delays," Chaos, Solitons and Fractals, vol. 35, no. 5, pp. 1003-1008, 2008.

[7] C. Peng, D. Yue, and Y.-C. Tian, "New approach on robust delay-dependent $H_{\infty}$ control for uncertain T-S fuzzy systems with interval time-varying delay," IEEE Transactions on Fuzzy Systems, vol. 17, no. 4, pp. 890-900, 2009.

[8] K.-W. Yu and C.-H. Lien, "Robust $H_{\infty}$ control for uncertain T-S fuzzy systems with state and input delays," Chaos, Solitons and Fractals, vol. 37, no. 1, pp. 150-156, 2008.

[9] L. Li and X. Liu, "New results on delay-dependent robust stability criteria of uncertain fuzzy systems with state and input delays," Information Sciences, vol. 179, no. 8, pp. 1134-1148, 2009.

[10] W.-J. Chang, C.-C. Ku, and Z.-G. Fu, "Robust and passive constrained fuzzy control for discrete fuzzy systems with multiplicative noises and interval time delay," Mathematical Problems in Engineering, vol. 2013, Article ID 159279, 12 pages, 2013.

[11] S. Ahmad, R. Majeed, K.-S. Hong, and M. Rehan, "Observer design for one-sided Lipschitz nonlinear systems subject to measurement delays," Mathematical Problems in Engineering, vol. 2015, Article ID 879492, 13 pages, 2015.

[12] L. Li and X. Liu, "New approach on robust stability for uncertain T-S fuzzy systems with state and input delays," Chaos, Solitons \& Fractals, vol. 40, no. 5, pp. 2329-2339, 2009.

[13] P. G. Park, J. W. Ko, and C. Jeong, "Reciprocally convex approach to stability of systems with time-varying delays," Automatica, vol. 47, no. 1, pp. 235-238, 2011.

[14] Y. S. Moon, P. Park, W. H. Kwon, and Y. S. Lee, "Delay-dependent robust stabilization of uncertain state-delayed systems," International Journal of Control, vol. 74, no. 14, pp. 1447-1455, 2001.

[15] Y. S. Lee, W. H. Kwon, and P. G. Park, "Authors reply: comments on delay-dependent robust $H_{\infty}$ control for uncertain systems with a state-delay," Automatica, vol. 43, no. 3, pp. 572-573, 2007.

[16] Z. Yang and Y.-P. Yang, "New delay-dependent stability analysis and synthesis of T-S fuzzy systems with time-varying delay,"
International Journal of Robust and Nonlinear Control, vol. 20, no. 3, pp. 313-322, 2010.

[17] D. H. Lee, J. B. Park, and Y. H. Joo, "A new fuzzy lyapunov function for relaxed stability condition of continuous-time takagi-sugeno fuzzy systems," IEEE Transactions on Fuzzy Systems, vol. 19, no. 4, pp. 785-791, 2011.

[18] L. A. Mozelli, R. M. Palhares, F. O. Souza, and E. M. A. M. Mendes, "Reducing conservativeness in recent stability conditions of T-S fuzzy systems," Automatica, vol. 45, no. 6, pp. 1580$1583,2009$.

[19] T. M. Guerra and L. Vermeiren, "LMI-based relaxed nonquadratic stabilization conditions for nonlinear systems in the Takagi-Sugeno's form," Automatica, vol. 40, no. 5, pp. 823-829, 2004.

[20] S. Zhou, J. Lam, and W. X. Zheng, "Control design for fuzzy systems based on relaxed nonquadratic stability and $H_{\infty}$ performance conditions," IEEE Transactions on Fuzzy Systems, vol. 15, no. 2, pp. 188-199, 2007.

[21] S. H. Kim, "Relaxation technique for a T-S fuzzy control design based on a continuous-time fuzzy weighting-dependent lyapunov function," IEEE Transactions on Fuzzy Systems, vol. 21, no. 4, pp. 761-766, 2013.

[22] S. H. Kim and P. Park, "Relaxed $H_{\infty}$ stabilization conditions for discrete-time fuzzy systems with interval time-varying delays," IEEE Transactions on Fuzzy Systems, vol. 17, no. 6, pp. 1441-1449, 2009.

[23] S. H. Kim, "Robust stability analysis of T-S fuzzy systems with interval time-varying delays via a relaxation technique," in Proceedings of the 8th IEEE International Conference on Automation Science and Engineering, pp. 829-832, Seoul, Korea, August 2012.

[24] K. Gu, "An integral inequality in the stability problem of timedelay systems," in Proceedings of the 39th IEEE Confernce on Decision and Control, pp. 2805-2810, Sydney, Australia, December 2000 .

[25] L. Li, X. Liu, and T. Chai, "New approaches on $H_{\infty}$ control of T-S fuzzy systems with interval time-varying delay," Fuzzy Sets and Systems, vol. 160, no. 12, pp. 1669-1688, 2009.

[26] J. An and G. Wen, "Improved stability criteria for time-varying delayed T-S fuzzy systems via delay partitioning approach," Fuzzy Sets and Systems, vol. 185, no. 1, pp. 83-94, 2011.

[27] C. G. Li, H. J. Wang, and X. F. Liao, "Delay-dependent robust stability of uncertain fuzzy systems with time-varying delays," IEE Proceedings-Control Theory and Applications, vol. 151, no. 4, pp. 417-421, 2004.

[28] C.-H. Lien, "Further results on delay-dependent robust stability of uncertain fuzzy systems with time-varying delay," Chaos, Solitons \& Fractals, vol. 28, no. 2, pp. 422-427, 2006.

[29] C. H. Lien, K. W. Yu, W. D. Chen, Z. L. Wan, and Y. J. Chung, "Stability criteria for uncertain Takagi-Sugeno fuzzy systems with interval time-varying delay," IET Control Theory and Applications, vol. 1, no. 3, pp. 764-769, 2007.

[30] F. Liu, M. Wu, Y. He, and R. Yokoyama, "New delay-dependent stability criteria for T-S fuzzy systems with time-varying delay," Fuzzy Sets and Systems, vol. 161, no. 15, pp. 2033-2042, 2010.

[31] A. Sala and C. Ariño, "Relaxed stability and performance conditions for Takagi-Sugeno fuzzy systems with knowledge on membership-function overlap," IEEE Transactions on Systems, Man, and Cybernetics, Part B: Cybernetics, vol. 37, no. 3, pp. 727732, 2007. 
[32] S. H. Kim and P. Park, " $H_{\infty}$ state-feedback-control design for discrete-time fuzzy systems using relaxation technique for parameterized LMI," IEEE Transactions on Fuzzy Systems, vol. 18, no. 5, pp. 985-993, 2010.

[33] M. Bernal, A. Sala, A. Jaadari, and T.-M. Guerra, "Stability analysis of polynomial fuzzy models via polynomial fuzzy Lyapunov functions," Fuzzy Sets and Systems, vol. 185, no. 1, pp. 5-14, 2011. 


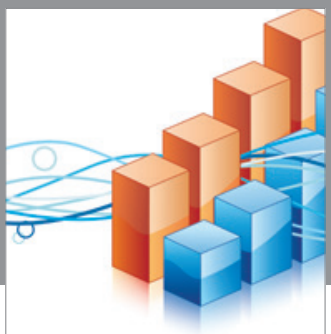

Advances in

Operations Research

vatem alat4

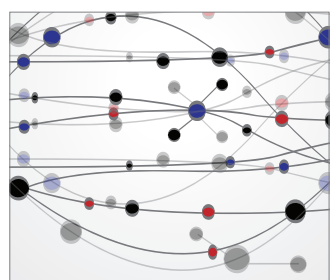

\section{The Scientific} World Journal
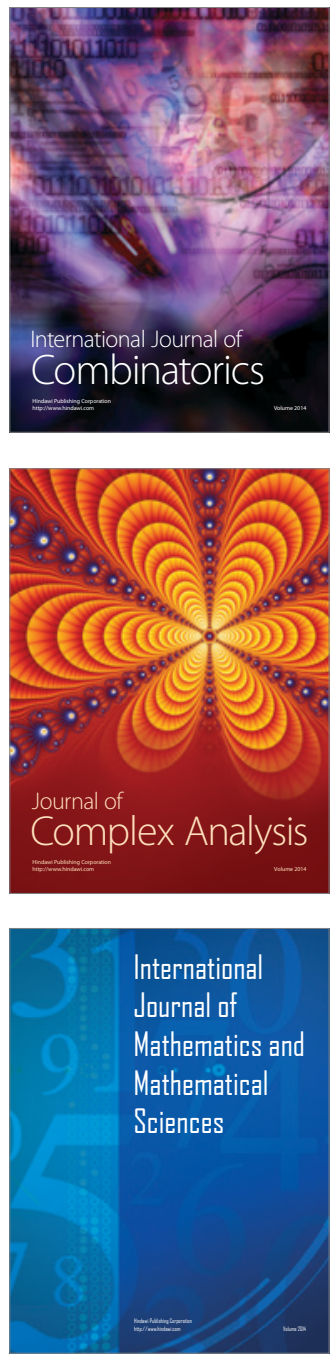
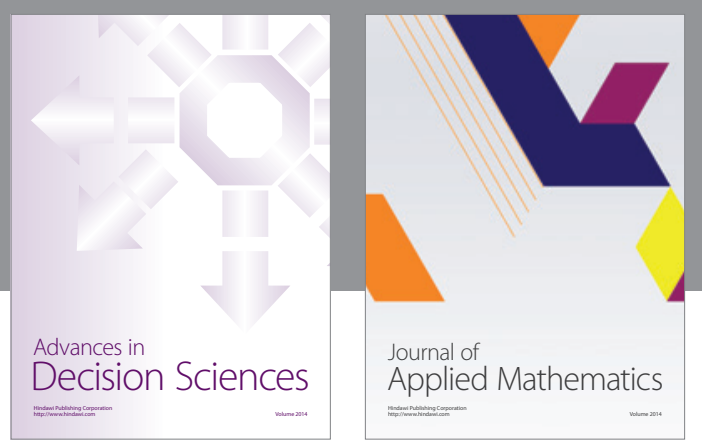

Algebra

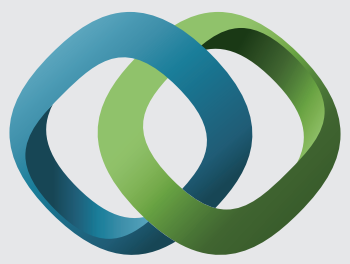

\section{Hindawi}

Submit your manuscripts at

http://www.hindawi.com
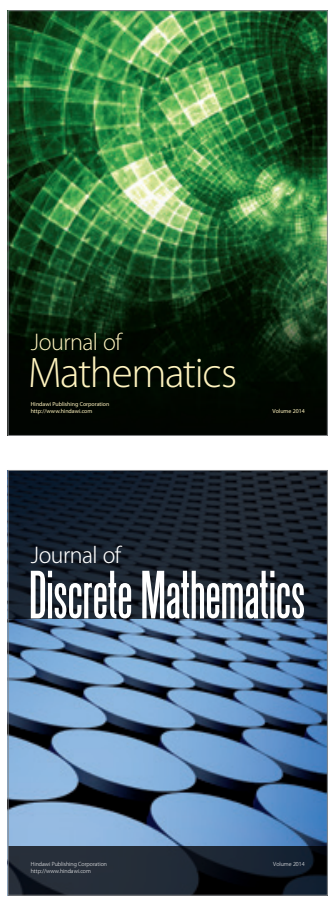

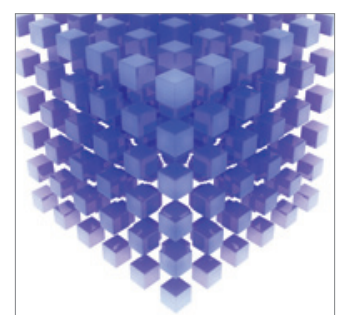

Mathematical Problems in Engineering
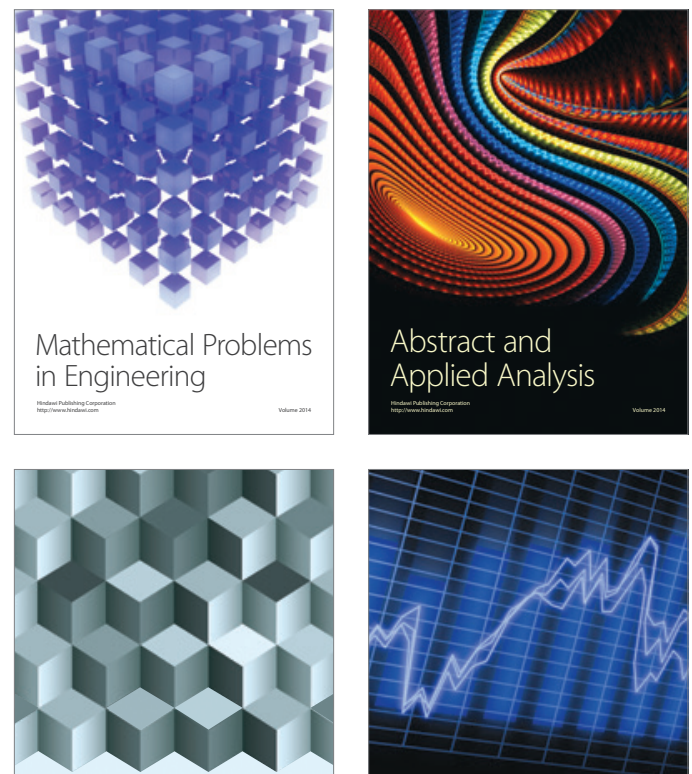

Journal of

Function Spaces

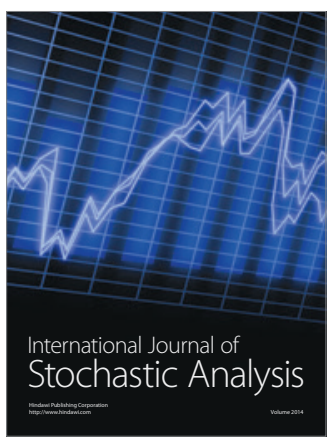

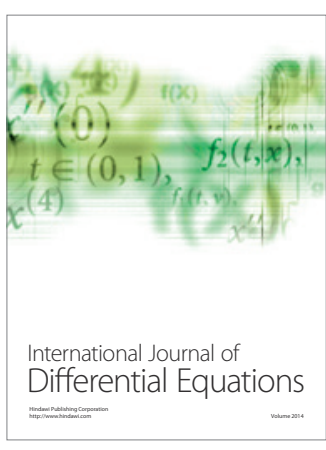
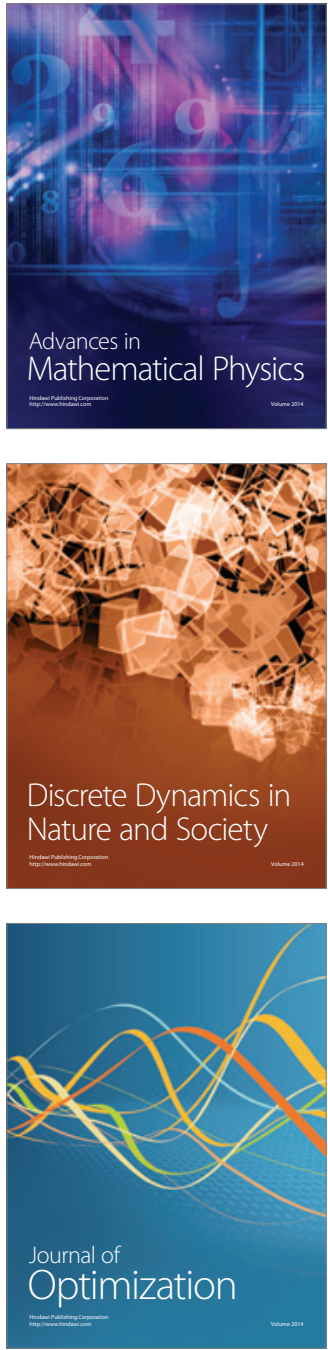